\title{
Role of Holes in Determining Resistivity and Hall Effect in Crystals of $\mathrm{YB}_{2} \mathrm{Cu}_{3} \mathrm{O}_{7-\delta}$ and $\mathrm{Bi}_{2} \mathrm{Sr}_{2} \mathrm{CaCu}_{2} \mathrm{O}_{8+\delta}$
}

\author{
Fred Wekesa Masinde \\ Department of Physical Sciences, South Eastern Kenya University, Kitui, Kenya \\ Email: fmasinde@seku.ac.ke
}

How to cite this paper: Masinde, F.W. (2019) Role of Holes in Determining Resistivity and Hall Effect in Crystals of $\mathrm{YB}_{2} \mathrm{Cu}_{3} \mathrm{O}_{7-\delta}$ and $\mathrm{Bi}_{2} \mathrm{Sr}_{2} \mathrm{CaCu}_{2} \mathrm{O}_{8+\delta}$. Open Access Library Journal, 6: e5663. https://doi.org/10.4236/oalib.1105663

Received: August 5, 2019

Accepted: August 24, 2019

Published: August 27, 2019

Copyright $\odot 2019$ by author(s) and Open Access Library Inc.

This work is licensed under the Creative Commons Attribution International License (CC BY 4.0).

http://creativecommons.org/licenses/by/4.0/

\section{(c) (i) Open Access}

\begin{abstract}
Electrical transport properties of superconducting materials are a widely studied area in superconductivity. In this study, the role of holes in determination of resistivity, Hall coefficient and Hall angle has been explored using a two-band model due to co-existence of holes and electrons in high- $T_{c}$ $\mathrm{YB}_{2} \mathrm{Cu}_{3} \mathrm{O}_{7-\delta}$ and $\mathrm{Bi}_{2} \mathrm{Sr}_{2} \mathrm{CaCu}_{2} \mathrm{O}_{8+\delta}$. The results obtained from this study show that hole resistivity $\left(\rho_{p}\right)$ decreases with increase in the ratio $u=\frac{\rho_{n}}{\rho_{p}}$ while
\end{abstract} the Hall coefficient $\left(R_{H}\right)$ is non-linearly dependent on the hole resistivity $\left(\rho_{p}\right)$. An increase in the hole scattering rate $\left(\gamma_{p}\right)$ causes a drop in the Hall scattering angle $\left(\theta_{H}\right)$.

\section{Subject Areas}

Applied Physics, Mechanical Engineering

\section{Keywords}

Holes, Resistivity, Hall Coefficient, Hall Number Density and Hall Scattering Angle

\section{Introduction}

Light elements such as $\mathrm{H}, \mathrm{Li}$ and $\mathrm{B}$ in metallic compounds have attracted considerable attention due to their potential superconducting properties [1] [2]. Within the weak-coupling BCS theory, high frequency phonons due to the presence of light mass atoms ensure a definite contribution to the BCS formula for the superconducting critical temperature $T_{c}$. Thus even a moderate electron-phonon Coupling can yield a sizeable $T_{c}$ [3] [4]. The discovery of super- 
conductivity in $\mathrm{MgB}_{2}$ [5], B-doped diamond [6], B-doped silicon [7] and other compounds [8] confirms this picture and extends it by showing that strong co-valent bonds between light atoms can provide a large contribution to the electronic density of states at the Fermi level, under proper doping conditions. In B-doped diamond, substitutional boron atoms provide hole-doping to the $\mathrm{sp}^{3}$ covalent bonds. The strong $\mathrm{C}-\mathrm{C}$ bonding allows the structure to remain stable even at high doping.

Nitrogen follows carbon in the first row of the periodic table and is characterized, in its elemental form, by a strong triple bond in the low pressure molecular phases, and by single covalent bonds in the non-molecular phase which is stable at pressures exceeding a mega-bar [9]. As a consequence, molecular phases are insulating and non-molecular phase is semi-conducting [10]. In fact, search for superconductivity in nitrogen-based systems requires the identification of compounds where covalent bonds between nitrogen atoms persist in a stable form in the presence of doping species. Experimental studies have shown that the high-frequency modes originating from the covalently bonded $\mathrm{N}_{2}$ units are strongly coupled to the electronic states at the Fermi surface and would give rise to a $T_{c}$ of about $1 \mathrm{~K}$ and the superconducting temperature can be greatly increased by hole-doping [11]. It is found that on changing the distance between nitrogen atoms in the N-N units along with the $x$ - $y$ polarized mode, the most relevant change in the Fermi surface (FS) is the migration of electrons from the bands that contain the hole pockets.

Study of the electronic density of states (DOS) of $\mathrm{OSN}_{2}$ suggests that hole-doping could further enhance $T_{c}$. Hole doping lowers the Fermi level towards a region of higher electronic density and would at the same time stiffen the N-N bonds by partially emptying the anti-bonding states below $E_{F}$. Consequences of hole-doping were studied with a hole-doping of 0.5 holes per unit cell in an alloy $\mathrm{OS}_{0.75} \mathrm{Re}_{0.25} \mathrm{~N}_{2}$ composition. It was found that the density of states at FS increases by about 2.4 times with respect to the undoped case. Consequently, the superconducting critical temperature for a doped $\mathrm{OSN}_{2}$ increases and $T_{c} \cong 4 \mathrm{~K}$.

Thus it was concluded that $\mathrm{OSN}_{2}$ is a superconductor and that its superconducting properties are connected to a strong coupling between the stretching modes of the covalently bonded $\mathrm{N}_{2}$ units with the electronic states at the Fermi level. This is similar to what has been observed in a number of boron- and carbon-based compounds, including $\mathrm{MgB}_{2}$. It is also predicted that the superconducting transition temperature can increase in $\mathrm{OSN}_{2}$ by doping it with holes. Experimentally, it can be achieved by synthesizing the nitride starting from a hole-doped OS alloy.

Another analysis that supports the role of holes in determining or obtaining the superconducting state is due to the co-existence of electrons and holes in high- $T_{c}$ superconducting materials superconducting materials in normal state [12]. DC resistivity and Hall Effect for cuprates based in $\mathrm{Y}$ or on $\mathrm{Bi} / \mathrm{Sr}$ find nearly linear-in- $\mathrm{T}$ temperature dependences in the resistivity (the symbol shown). The 
hole-number density $n_{H}$ is given by:

$$
n_{H}=\frac{1}{e\left|R_{H}\right|}
$$

where $R_{H}$ is the Hall coefficient and $e$ is the elementary charge (positive by convention) [13]. Discovery of these results in $\mathrm{YBa}_{2} \mathrm{Cu}_{3} \mathrm{O}_{7-\delta}$ prompted the early speculations on the electron hole co-existence in the $a-b$ plane transport to explains the near-linear temperature dependence of $n_{H}$, ascribing the positive and temperature dependence of $R_{H}$ to dominance of transport by holes over electrons by differing velocities.

Various theoretical models have been proposed to explain the DC Hall Effect based on electron-hole interpretation. The temperature dependence in $R_{H}$ is assumed to arise from temperature-dependent anisotropy of the scattering length $l_{R}=V_{R}+\tau_{R}$ (velocity-scattering time product) around the Fermi contour [14]. Another approach was when the energy levels $E$ above the saddle point $E_{s}$ of a single $\mathrm{CuO}_{2}$ band are treated as a hole-like and levels $E<E_{s}$ as electron-like [14]. The temperature dependence of $R_{H}$ is then contained in an ad-hoc model for energy dependence in scattering anisotropy. However introducing two carrier bands, one of electrons and one of holes, one can study the temperature dependence in $R_{H}$, and the resistivity and hall effect can be modelled with two band carriers; holes and electrons; and also the relationship to the superconducting state can be understood.

\subsection{Superconducting States}

One of the puzzles of high- $T_{c}$ superconductivity is that the residual ac conductivity at low temperature in the limit of zero frequency cannot be explained for moderate impurity scattering (within the context of either the d-wave or s-wave pairing), and suggests the presence of a component with substantial pair breaking in the superconducting state [15]. Allowing for co-existence of electrons and holes bands can solve this problem. A large number of attempts [16]-[23] were made to study the low-temperature thermal properties, Hall effect and resistivity of superconducting materials and these studies pointed to the presence of electrons co-existing with the superconducting condensate of holes and maintained in metastable states via strong scattering the normal state resistivity and Hall effect in $\mathrm{Bi}_{2} \mathrm{Sr}_{2} \mathrm{CaCu}_{2} \mathrm{O}_{8+\delta}$ and $\mathrm{YBa}_{2} \mathrm{Cu}_{3} \mathrm{O}_{7-\delta}$ could be modelled by reducing the band structures of these high- $T_{c}$ compounds to two components comprising of holes and electrons in co-existence [12]. It provided explanation for temperature dependence in the Hall coefficient and the presence of electrons due to existence of negative Hall mobility intercept $\mu_{H}^{-1}(T \rightarrow 0)<0$. A large number of samples of $\mathrm{YB}_{2} \mathrm{Cu}_{3} \mathrm{O}_{7-\delta}$ were studied to study the DC Hall effect and to confirm the presence of at least two bands with oppositely signed Hall coefficients. In the analysis it was found that taking the Hall number densities of electrons and holes to be equal is sufficient for agreement with the experiment [12]. Thus, the theoretical and experimental studies conducted so far sufficiently confirm the co-existence 
of holes and electrons in high- $T_{c}$ superconductors and such a concept can be used to study some of the properties of superconductors such as resistivity and Hall Effect.

\subsection{Theoretical Derivations}

The Lorentz force, $\boldsymbol{F}$ acting on an electron of charge $e$ moving with velocity $\boldsymbol{V}$ perpendicular to the applied magnetic field $\boldsymbol{B}$, with electric field $\boldsymbol{E}$, acting on the electron is:

$$
\boldsymbol{F}=e(\boldsymbol{E}+\boldsymbol{v} \times \boldsymbol{B})
$$

when the downward force is balanced by the upward force of developed transverse field, the resultant force is zero. Thus,

$$
\boldsymbol{F}_{y}=0=e\left(\boldsymbol{E}_{y}+\boldsymbol{v}_{x} \boldsymbol{B}_{z}\right)
$$

Or

$$
\boldsymbol{E}_{y}=\boldsymbol{v}_{x} \boldsymbol{B}_{z}
$$

If $d$ is the width of the metal bar, then the potential difference, $U$, developed across the bar is

$$
U=\boldsymbol{E}_{y} d=\boldsymbol{v}_{x} \boldsymbol{B}_{z} d
$$

Now, the current density, $\boldsymbol{j}_{x}$, along the $x$-axis is

$$
\boldsymbol{j}_{x}=N e \boldsymbol{v}_{x}
$$

where $N$ is the number of charge carriers per $\mathrm{cm}^{3}$ or it is also called Hall number density. We can now write,

$$
\frac{1}{N e}=\frac{\boldsymbol{E}_{y}}{\boldsymbol{j}_{x} \boldsymbol{B}_{z}}=R_{H}
$$

where $R_{H}$ is called the Hall coefficient or constant and it is negative for most metals where the current is carried by the electrons that have negative charge. For some metals like $\mathrm{Be}, \mathrm{Zn}, \mathrm{Cd} ; R_{H}$ is positive indicating that the current is also carried by a positive charge.

\subsection{Two-Band Model: Resistivity and Hall Coefficient}

In the normal state, the electrical transport properties in the $a-b$ plane of $\mathrm{YBa}_{2} \mathrm{Cu}_{3} \mathrm{O}_{7-\delta}$ are consistent with a two-band model comprising electrons and holes as charge carriers of opposite signs. In the distorted $\mathrm{OSN}_{2}$ crystal, the most relevant change in the Fermi surface (FS) is the migration of electrons from the bands that contain the hole pockets to the bands that contain the electron pockets. Thus, the electron-hole co-existence will be relevant in the study of resistivity and Hall coefficient.

Now, the electrical resistivity $\rho$ can be modelled with parallel transport by electrons and holes. Writing the electron resistivity as $\rho_{n}$ and that of holes as $\rho_{p}$, the net resistance $R$ of the parallel resistance circuit can be written as

$$
\frac{1}{R}=\frac{1}{R_{e}}+\frac{1}{R_{n}}
$$


Or

$$
R=\left(R_{e}^{-1}+R_{n}^{-1}\right)^{-1}
$$

And hence,

$$
\rho=\left(\rho_{n}^{-1}+\rho_{p}^{-1}\right)^{-1}
$$

We can now apply the two-band model to the specific cases of the DC and AC Hall Effect.

\subsection{Hall Effect Coefficient}

If $R_{H n}$ is the Hall coefficient for electrons and $R_{H p}$ is the Hall coefficient for holes, then using the two-band model, the Hall coefficient $R_{H}$ of the material can be written as

$$
R_{H}=\frac{\rho_{n}^{-2} R_{H n}+\rho_{p}^{-2} R_{H p}}{\left(\rho_{n}^{-1}+\rho_{p}^{-1}\right)^{2}}
$$

where $R_{H n}$ is to be negative, $R_{H p}$ is to be positive and both quantities are to be temperature-independent constants of the materials under consideration. To apply Equation (8) to describe the measured Hall coefficient, it should be understood that the Hall scattering rate is a function of the hole and electron resistivity components which determine the scattering rates for electrical transport. The model takes into account that $\rho(T)$ and $R_{H}(T)$ can vary with $T$ in the normal state and $R_{H}(T)$ is positive. In fact we cannot assume any definite functional form for $\rho(T)$ and $R_{H}(T)$ since the temperature dependences are not identical among various types of specimens. Moreover, in the two-band behaviour, Hall Effect analysis does not convey information on carrier concentrations.

We define $T$-dependent variables as

$$
w=\frac{R_{H}}{R_{H p}} \text { and } u=\frac{\rho_{n}}{\rho_{p}}
$$

And $T$-independent constant as,

$$
r=\frac{R_{H n}}{R_{H p}}
$$

Between Equation (3) and Equation (4) we can write

$$
\rho=\frac{1}{\rho_{n}^{-1}+\rho_{p}^{-1}}=\frac{1}{\frac{1}{\rho_{n}}+\frac{u}{\rho_{n}}}=\frac{\rho_{n}}{1+u}
$$

Or

$$
\rho_{n}=\rho(1+u)
$$

Similarly, using Equation (4) to eliminate $\rho_{n}$ from Equation (3), we get

$$
\rho_{p}=\rho\left(\frac{1+u}{u}\right)
$$


Now we can write

$$
R_{H}=\frac{\frac{R_{H n}}{\rho_{n}^{2}}+\frac{R_{H p}}{\rho_{p}^{2}}}{\left(\frac{1}{\rho_{n}}+\frac{1}{\rho_{p}}\right)^{2}}=\frac{\rho_{p}^{2} R_{H n}+\rho_{n}^{2} R_{H p}}{\rho_{p}^{2} \rho_{n}^{2}\left(\frac{\rho_{p}+\rho_{n}}{\rho_{p} \rho_{n}}\right)^{2}}=\frac{\rho_{p}^{2} R_{H n}+\rho_{n}^{2} R_{H p}}{\left(\rho_{p}+\rho_{n}\right)^{2}}
$$

Dividing both sides by $R_{H p}$ gives

$$
\frac{R_{H}}{R_{H p}}=\frac{\rho_{p}^{2} \frac{R_{H n}}{R_{H p}}+\rho_{n}^{2}}{\left(\rho_{p}+\rho_{n}\right)^{2}} \text { or } w=\frac{\rho_{p}^{2}(-r)+\rho_{n}^{2}}{\left(\rho_{p}+\rho_{n}\right)^{2}}
$$

Substituting for $\rho_{p}$ and $\rho_{n}$ using Equation (12) and Equation (13) into Equation (15), we have

$$
\frac{R_{H}}{R_{H p}}=\frac{-r \rho^{2}\left(\frac{1+u}{u}\right)^{2}+\rho^{2}(1+u)^{2}}{\rho^{2}\left(\frac{1+u}{u}+(1+u)\right)^{2}}=\frac{-r(1+u)^{2}+u^{2}(1+u)^{2}}{((1+u)+u(1+u))^{2} \frac{1}{u^{2}}} ?
$$

Thus,

$$
w=\frac{(1+u)^{2}\left(u^{2}-r\right) u^{2}}{(1+u)^{4}}=\frac{\left(u^{2}-r\right) u^{2}}{(1+u)^{2}}
$$

Equation (16) leads to the following information and analysis. If $R_{H}$ is positive, then $w$ is positive since $R_{H p}$ is positive. Equation (16) then gives that $u^{2}>r$. If the two Hall coefficient components are equal, then $r=1$ and then we can define a single Hall constant as $R_{H 0}=-R_{H n}=R_{H p}$. FROM Equation (8) it is clear that the quantities $\left(\frac{\rho_{n}}{\rho_{p}}\right)^{2}$ and $\left(\frac{R_{H n}}{R_{H p}}\right)$ are strongly correlated (almost linearly) so that the cases for $r \neq 1$ can be obtained by scaling $\left(\frac{\rho_{n}}{\rho_{p}}\right)$. Now for $r=1$ and $u^{2}>r\left(u^{2}>1\right.$ or $\left.u>1\right)$ it will mean that $\rho_{n}(T)>\rho_{p}(T)>\rho(T)$. In such a case, $\rho_{n}(T)$ will have a weaker temperature dependence than $\rho_{p}(T)$ ( $\left.\rho_{p}(T) \cong \rho_{0} \mathrm{e}^{-\frac{\epsilon}{k T}}\right)$. The simplest model for temperature dependence of electronic component of resistivity $\rho_{n}(T)$ is a linear function of temperature, $T$, containing two adjustable parameters, i.e.

$$
\rho_{n}(T)=a_{1}+b_{1} t
$$

where $t=\frac{T}{T_{0}}$ is a dimensionless variable. The temperature dependent hole resistivity $\rho_{p}(T)$ is determined from the solution of Equation (7) since $\rho_{n}(T)$ will be known from Equation (18). This gives $\rho_{p}(T)$ as

$$
\rho_{p}(T)=\left(\rho^{-1}(T)-\rho_{n}^{-1}(T)\right)^{-1}
$$




\subsection{AC Hall Effect: Hall Angle and Scattering Rates}

The Hall angle components $\left(\theta_{H_{p, n}}\right)$ are defined as

$$
\theta_{H_{p, n}}=\frac{\omega_{H_{p, n}}}{\gamma_{H_{p, n}}}
$$

where $\omega_{H_{p, n}}=\frac{\boldsymbol{B}}{m_{p, n}}$ are the Hall frequencies and $\gamma_{H_{p, n}}$ are the scattering rates. $\boldsymbol{B}$ is the applied magnetic field. For $\boldsymbol{B}<10 \mathrm{~T}$, the Hall angle in High- $T_{c}$ superconductors is low compared to unity, so that $\theta_{H}$ can be written as

$$
\theta_{H}=\frac{R_{H} \boldsymbol{B}}{\rho}
$$

Now, using Equation (8) and Equation (9),

$$
\begin{gathered}
\rho=\rho_{n}^{-1}+\rho_{p}^{-1} ; R_{H}=\frac{\rho_{n}^{-2} R_{H n}+\rho_{p}^{-2} R_{H p}}{\rho_{n}^{-1}+\rho_{p}^{-1}} \\
\theta_{H}=\text { Hall angle }=R_{H}\left(\frac{\boldsymbol{B}}{\rho}\right)=\frac{\rho_{n}^{-2} R_{H n}+\rho_{p}^{-2} R_{H p}}{\rho_{n}^{-1}+\rho_{p}^{-1}} \boldsymbol{B}
\end{gathered}
$$

In the model considered (two-band model), the resistivity components are expressed in terms of the scattering rates as,

$$
\rho_{p, n}=\frac{m_{p, n} \gamma_{p, n}}{n_{p, n} e^{2}}
$$

where $m_{p, n}$ are the effective masses and $n_{p, n}$ are the Hall carrier densities of holes and electrons. The expressions for the whole angle can now be written in the form

$$
\theta_{H}=\frac{\frac{\theta_{H p}}{\rho_{p}}+\frac{\theta_{H n}}{\rho_{n}}}{\left(\frac{1}{\rho_{n}}+\frac{1}{\rho_{p}}\right)}=\frac{\rho_{n} R_{H p}+\rho_{p} R_{H n}}{\rho_{p}+\rho_{n}}
$$

Substituting Equation (23) into Equation (24) gives

$$
\theta_{H}=\frac{\frac{\omega_{H, p} m_{n} \gamma_{n}}{n_{p} \gamma_{p}}+\frac{\omega_{H, n} m_{p} \gamma_{p}}{n_{n} \gamma_{n}}}{\frac{m_{p} \gamma_{p}}{n_{p}}+\frac{m_{n} \gamma_{n}}{n_{n}}}
$$

We now introduce two parameters, one for the ratio of masses $r_{m}=\frac{m_{n}}{m_{p}}$ and the ratio of carrier densities $r_{n}=\frac{n_{n}}{n_{p}}$ and re-write Equation (25) as

$$
\theta_{H}=\left(\frac{\omega_{H, 0}}{m_{p} / m_{0}}\right) \frac{\frac{r_{m} \gamma_{n}}{r_{N} \gamma_{p}}-\frac{\gamma_{p}}{r_{N} \gamma_{n}}}{\gamma_{p}+\frac{r_{m} \gamma_{n}}{r_{N}}}
$$


where $\omega_{H, 0}=\frac{e \boldsymbol{B}}{m_{0}}$ and $m_{0}$ is the rest mass of the electron and the sign of $\omega_{H, n}$ is negative for the electron component since the charge on the electron is $(-e)$.

\section{Results and Discussion}

Equation (12) and Equation (13) have been used to plot the graph of resistivity ( $\rho$ ) as a function of $u=\frac{\rho_{n}}{\rho_{p}}$ in Figure 1 for the temperature range $95 \mathrm{~K}$ to 190 $\mathrm{K}$ experimental results have shown that $\rho=130 \mu \Omega \cdot \mathrm{cm}$ and $u=1.49$ to 2.77 [24]. The plot in Figure 1 shows a linear dependence of electron resistivity on $u$ and a nonlinear dependence of hole resistivity on $u$. The values of $\rho_{n}$ and $\rho_{p}$ over the temperature range $95 \mathrm{~K}$ to $190 \mathrm{~K}$ are $323 \mu \Omega \cdot \mathrm{cm}$ to $490 \mu \Omega \cdot \mathrm{cm}$ and $217 \mu \Omega \cdot \mathrm{cm}$ to $176 \mu \Omega \cdot \mathrm{cm}$ respectively. This means that as temperature increases from $95 \mathrm{~K}$ to $190 \mathrm{~K}$, electron resistivity increases while hole resistivity deceases.

Equation (14) has been used to plot the graph of Hall coefficient $\left(R_{H}\right)$ as a function of hole resistivity $\left(\rho_{p}\right)$ in Figure 2. For a temperature range of $95 \mathrm{~K}$ to $190 \mathrm{~K}$, experimental results reveal that $R_{H n}=R_{H p}=0.01 ; \rho_{n}=390 \mu \Omega \cdot \mathrm{cm}$ and $\rho_{p}=100 \mu \Omega \cdot \mathrm{cm}$ to $250 \mu \Omega \cdot \mathrm{cm}$ [25]. The graph in Figure 2 shows that there is a non-linear dependence by Hall coefficient on the hole resistivity. In the temperature under consideration, $R_{H}$ varies between 0.0068 to 0.0052 . Clearly, an increase in the hole resistivity leads to a drop in the Hall coefficient.

Equation (25) has been used to plot the graph of Hall scattering angle $\left(\theta_{H}\right)$ as a function of hole scattering rate $\left(\gamma_{p}\right)$ in Figure 3. Experimentally, $\frac{\omega_{H 0}}{m_{p} / m_{0}}=0.146$; $r_{M}=1.30 ; r_{N} \approx 1 ; \frac{r_{M} \gamma_{n}}{r_{N} \gamma_{p}}=2.13 ; \gamma_{n}=235 \mathrm{~cm}^{-1}$ and $\gamma_{p}=112 \mathrm{~cm}^{-1}$ to $200 \mathrm{~cm}^{-1}$

[25]. The graph in Figure 2 shows that there is a non-linear dependence by Hall

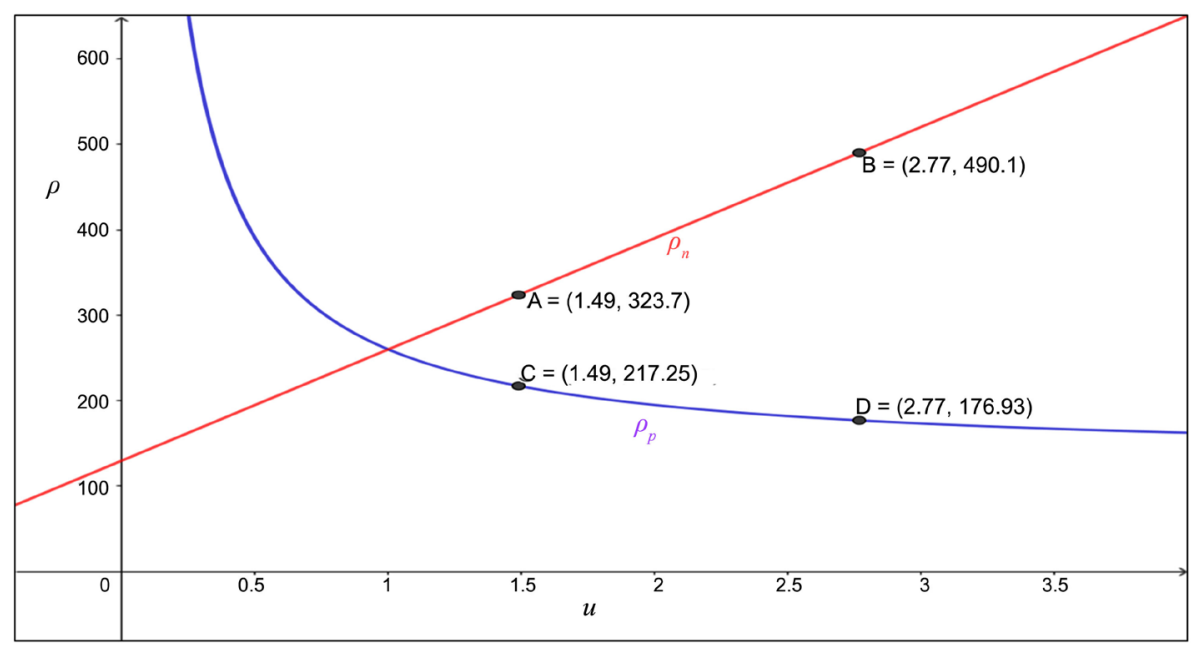

Figure 1. A graph of resistivity $(\rho)$ as a function of $u=\frac{\rho_{n}}{\rho_{p}}$. 


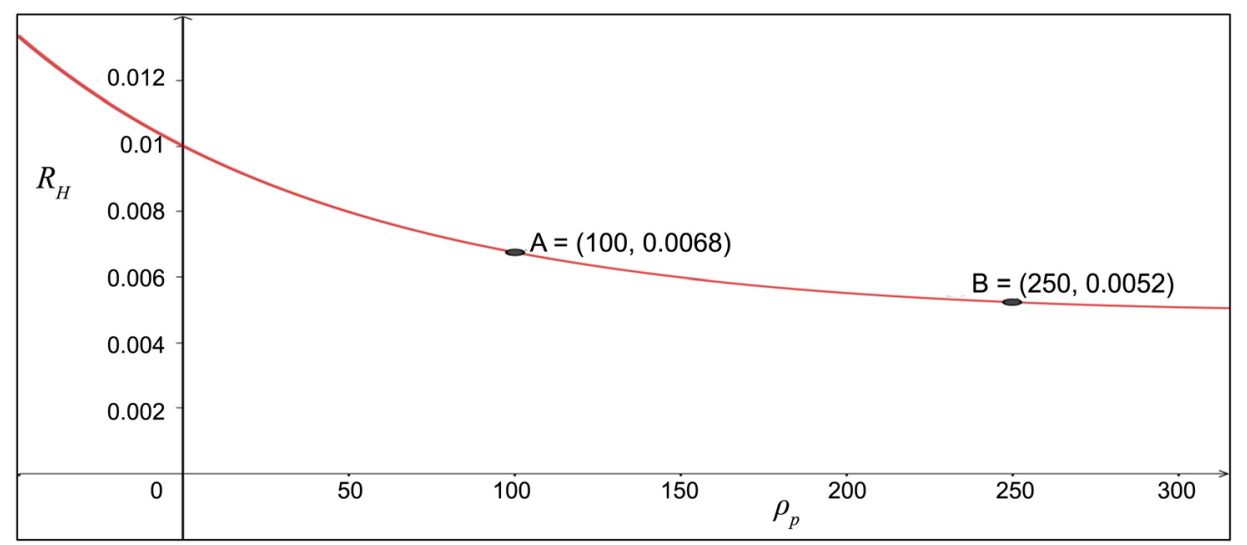

Figure 2. A graph of Hall coefficient ( $R_{H}$ ) as a function of hole resistivity $\rho_{p}$.

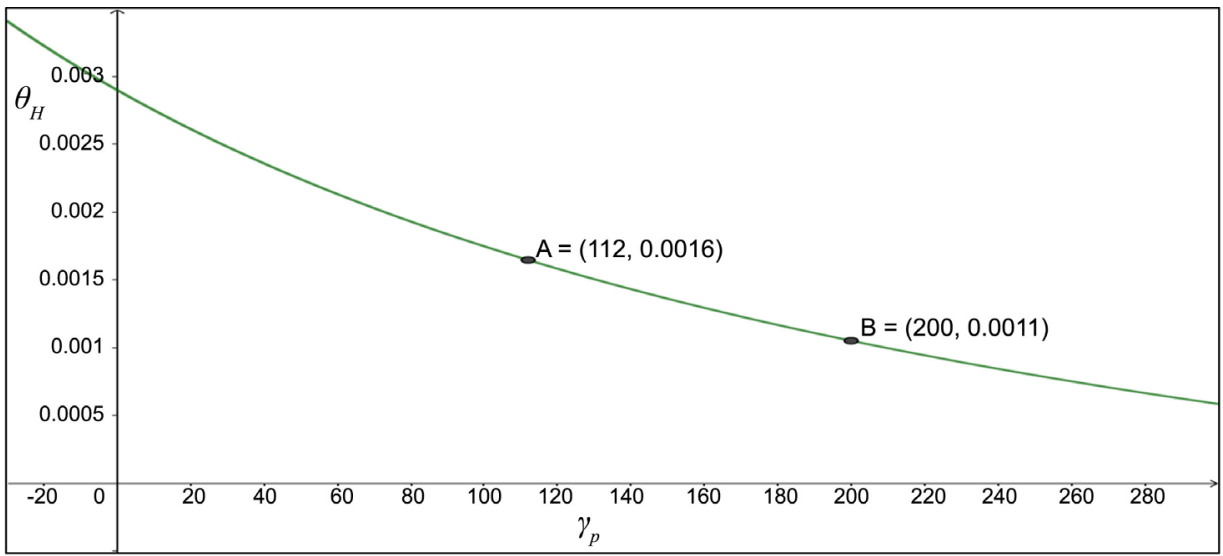

Figure 3. A graph of Hall scattering angle $\left(\theta_{H}\right)$ as a function of hole scattering rate $\gamma_{p}$.

coefficient on the hole resistivity. In the temperature under consideration, $R_{H}$ varies between 0.0016 to 0.0011 . An increase in the hole scattering rate lowers the Hall scattering angle.

Figure 1 shows that hole resistivity with increase in $u$ electron resistivity is linearly dependent on $u$. These two observations imply that as the temperature increases, hole resistivity decreases while the electron resistivity decreases. When the temperature decreases up to $T_{\mathcal{O}}$ electron resistivity fall to almost zero and the material turns into a superconductor. In Figure 2, the Hall coefficient decreases with hole resistivity while the Hall scattering angle in Figure 3 decreases with increase in Hall scattering rate.

\section{Conclusion}

In summary, it has been found that as the temperature rises, above the critical temperature of a superconductor, the hole resistivity reduces while the electron resistivity increases. As the hole resistivity increases towards the critical temperature from the temperature $T>T_{c}$, a non-linear drop in the Hall coefficient is recorded. Finally, the dependence of the Hall scattering angle on the scattering rate is found to be nonlinear. 


\section{Acknowledgements}

I am grateful to Professor K. M. Khanna in the Department of Physics in the University of Eldoret for his professional input in this research. I also wish to thank Mr. A. Mukubwa for support and linking me to the Open Access Library Journal.

\section{Conflicts of Interest}

The author declares no conflicts of interest regarding the publication of this paper.

\section{References}

[1] Chaudhari, P., Collins, R.T., Freitas, P., Gambino, R.J., Kirtley, J.R., Koch, R.H., Laibowitz, R.B., LeGoues, F.K., McGiure, T.R., Penney, T., Schlesinger, Z., Segmuller, A.P., Fonner, S. and Mc Niff, E.J. (1987) Properties of Epitaxial Films of $\mathrm{YBa}_{2} \mathrm{Cu}_{3} \mathrm{O}_{7-\delta .}$. Physical Review B, 36, 8903(R). https://doi.org/10.1103/PhysRevB.36.8903

[2] Penney, T., Von Molnar, S., Kaiser, D., Holtzberg, F. and Kleinsasser, A.W. (1988) Strongly Anisotropic Electrical Properties of Single-Crystal $\mathrm{YBa}_{2} \mathrm{Cu}_{3} \mathrm{O}_{7-\mathrm{x}}$. Physical Review B, 38, 2918(R). https://doi.org/10.1103/PhysRevB.38.2918

[3] Eagles, D.M. (1989) Concentrations and Mobilities of Holes and Electrons in a Crystal of a 90K Oxide Superconductor from Analysis of Ab-Plane Resistivity and Hall Data. Solid State Communications, 69, 229-234. https://doi.org/10.1016/0038-1098(89)90840-5

[4] Fiory, A.T. and Grader, G.S. (1988) Extraordinary Hall Effect in [Math Processing Error] Superconductors. Physical Review B, 38, 9198. https://doi.org/10.1103/PhysRevB.38.9198

[5] Chien, T.R., Brawner, D.A., Wang, Z.Z. and Ong, N.P. (1991) Unusual 1/[Math Processing Error] temperature dependence of the Hall conductivity in [Math Processing Error] [Math Processing Error] [Math Processing Error]. Physical Review $B, 43,6242$ (R). https://doi.org/10.1103/PhysRevB.43.6242

[6] Chien, T.R., Wang, Z.Z. and Ong, N.P. (1991) Effect of Zn Impurities on the Normal-State Hall Angle in Single-Crystal [Math Processing Error][Math Processing Error] [Math Processing Error] [Math Processing Error]. Physical Review Letters, 67, 2088. https://doi.org/10.1103/PhysRevLett.67.2088

[7] Rice, J.P., Giapintzakis, J., Ginsberg, D.M. and Mochel, J.M. (1991) Hall Effect above [Math Processing Error] in Untwinned Single-Crystal [Math Processing Error] [Math Processing Error][Math Processing Error]: Normal-State Behavior and Superconducting Fluctuations. Physical Review B, 44, 10158. https://doi.org/10.1103/PhysRevB.44.10158

[8] Harris, J.M., Yan, Y.F. and Ong, N.P. (1992) Experimental Test of the [Math Processing Error] Law for the Hall Angle from [Math Processing Error] to $500 \mathrm{~K}$ in Oxygen-Reduced [Math Processing Error][Math Processing Error][Math Processing Error] Crystals. Physical Review B, 46, 14293(R). https://doi.org/10.1103/PhysRevB.46.14293

[9] Jiang, W., Peng, J.L., Hagen, S.J. and Green, L.R. (1992). Hall-Effect Studies of $\mathrm{Y}_{1-\mathrm{x}} \mathrm{Pr}_{\mathrm{x}} \mathrm{Ba}_{2} \mathrm{Cu}_{3} \mathrm{O}_{7}$ Crystals. Phys. Rev. B, 46, 8694(R).

[10] Xiao, G., Xiong and Cieplak, M.Z. (1992) Universal Hall Effect in [Math Processing 
Error][Math Processing Error][Math Processing Error][Math Processing Error] [Math Processing Error] Systems (A = Fe, Co, Ni, Zn, Ga). Physical Review $B$, 46, 8687(R). https://doi.org/10.1103/PhysRevB.46.8687

[11] Carrington, A., Walker, D.J.C., Mackenzie, A.P. and Cooper, J.R. (1993) Hall Effect and Resistivity of Oxygen-Deficient [Math Processing Error][Math Processing Error] [Math Processing Error] Thin Films. Physical Review B, 48, 13051. https://doi.org/10.1103/PhysRevB.48.13051

[12] Wuyts, B., Osquilquil, E., Maenhoudt, M., Libbrecht, S., Gao, Z.X. and Bruynseraede, Y. (1993) Influence of the Oxygen Content on the Normal-State Hall Angle in [Math Processing Error][Math Processing Error][Math Processing Error] Films. Physical Review B, 47, 5512(R). https://doi.org/10.1103/PhysRevB.47.5512

[13] Xiong, Xiao, G. and Wu, X.D. (1993) Hall Angle in [Math Processing Error] [Math Processing Error][Math Processing Error] Epitaxial Films: Comparison between Oxygen Reduction and Pr Doping. Physical Review B, 47, 5516(R). https://doi.org/10.1103/PhysRevB.47.5516

[14] Lang, W., Hein, G., Schwab, Wang, X.Z. and Bauerle, D. (1994) Paraconductivity and Excess Hall Effect in Epitaxial [Math Processing Error][Math Processing Error][Math Processing Error] Films Induced by Superconducting Fluctuations. Physical Review B, 49, 4209. https://doi.org/10.1103/PhysRevB.49.4209

[15] Hpfengartner, R., Lippert, M., Dorsch, W., Kreiselmeyer, G. and Saemann-Ischengo, G. (1994) Transport Properties of Oxygen-Deficient $\mathrm{YBa}_{2} \mathrm{Cu}_{3} \mathrm{O}_{7-\mathrm{x}}$ Thin Films. Journal of Superconductivity, 7, 319-322. https://doi.org/10.1007/BF00724561

[16] Hofmann, L., Karl, H. and Samwer, K. (1994) Normal-State Hall Effect and Resistivity Measurements of $\mathrm{YBa}_{2} \mathrm{Cu}_{3} \mathrm{O}_{\mathrm{x}}$-Films with Different Oxygen Content. Zeitschrift für Physik B, 95, 173-180. https://doi.org/10.1007/BF01312189

[17] Hwang, H.Y., Batlogg, B., Tagaki, H., Kao., H.L., Kwo, R.J., Cava, R.J., Krajewski, J.J. and Peck, W.F. (1994) Scaling of the Temperature Dependent Hall Effect in $\mathrm{La}_{2-\mathrm{x}} \mathrm{Sr}_{\mathrm{x}} \mathrm{CuO}_{4}$. Physical Review Letters, 72, 2636.

https://doi.org/10.1103/PhysRevLett.72.2636

[18] Jin, R. and Ott, H.R. (1998) Hall Effect of $\mathrm{YBa}_{2} \mathrm{Cu}_{3} \mathrm{O}_{7-\delta}$ Single Crystals. Physical Review $B$, 57, 13872. https://doi.org/10.1103/PhysRevB.57.13872

[19] Yamada, T., Motohashi, M., Kobayashi, H., Fujita, T., Shimoyama, J. and Kishio, K. (1999) Resistivity Anisotropy and Hall Coefficient of Pb-Doped Bi2212 Single Crystals. Journal of Low Temperature Physics, 117, 1217-1221. https://doi.org/10.1023/A:1022527818911

[20] Ando, Y. and Murayama, T. (1999) Nonuniversal Power Law of the Hall Scattering Rate in a Single-Layer Cuprate $\mathrm{Bi}_{2} \mathrm{Sr}_{2-\mathrm{x}} \mathrm{La}_{\mathrm{x}} \mathrm{CuO}_{6}$. Physical Review B, 60, 6991(R). https://doi.org/10.1103/PhysRevB.60.R6991

[21] Konstaninovic, Z., Li, Z.Z. and Rffy, H. (2000) Temperature Dependence of the Hall Effect in Single-Layer and Bilayer $\mathrm{Bi}_{2} \mathrm{Sr}_{2} \mathrm{Can}_{-1} \mathrm{Cu}_{\mathrm{n}} \mathrm{O}_{\mathrm{y}}$ Thin Films at Various Oxygen Contents. Physical Review B, 62, 11989(R).

[22] Tozer, S.W., Kleinsasser, A.W., Penny, T., Kaiser, D. and Holtzberg, F. (1987) Measurement of Anisotropic Resistivity and Hall Constant for Single-Crystal $\mathrm{YBa}_{2} \mathrm{Cu}_{3} \mathrm{O}_{7-\mathrm{x}}$. Physical Review Letters, 59, 1768.

https://doi.org/10.1103/PhysRevLett.59.1768

[23] Markiewicz, R.S. (1988) Simple Model for the Hall Effect in $\mathrm{YBa}_{2} \mathrm{Cu}_{3} \mathrm{O}_{7-\delta}$. Physical Review B, 38, 5010. https://doi.org/10.1103/PhysRevB.38.5010 
[24] Harshman, D.R., Dow, J.D. and Fiory, A.I. (2011) Theory of High-Tc Superconductivty: Transition Temperature. arXiv: cond-mat/1202.0306

[25] Grayson, M., Rigal, L.B., Schmadel, D.C., Drew, H.D. and Kung, J. (2000) Spectral Measurement of the Hall Angle Response in Normal State Cuprate Superconductors. Physical Review Letters, 89, Article ID: 037003. 